\title{
Functional State Spaces and their Formation in Systems from Biological Organisms to the Physical Universe
}

\author{
Andy E. Williams ${ }^{1 *}$ \\ ${ }^{1}$ Nobeah Foundation, Nairobi, Kenya \\ *awilliams@nobeahfoundation.org
}

\begin{abstract}
This paper explores how the emerging science of Human-Centric Functional Modeling or HCFM provides a universal approach to modeling systems that is hypothesized to maximize human capacity to understand and navigate the complexity of systems, and how it facilitates a kind of biomimicry in which the human organism is represented in terms of abstract mathematical spaces that can be used to define simple expressions to represent properties like "complexity" for human systems like cognition, where the same spaces can be used to represent other systems, including the entire physical universe, so that the underlying equivalence of the representations allows the same mathematical expressions to define the same properties where applicable for these very different systems, and therefore allows deep insights to potentially be gained about these systems through looking inward to observe how one's own cognition functions from one's first person experience. From this perspective the properties governing the evolution of life in its functional state space might also govern the formation of the universe in its own functional state space. Other significant potential benefits are that in defining behavior in terms of mathematical spaces enables all the mathematical disciplines that apply to such spaces (e.g. functor theory, category theory, process theory) to be used to understand and navigate the relationships between concepts described in those spaces. Another is that in providing a self-contained representation of the human meaning of any entity, including of any region in the physical universe, Human-Centric Functional Modeling potentially defines the first complete semantic representation of concepts, physical objects, or any other entities represented in a functional state space. When applied to the physical universe this implies that all theoretical or experimental data can be stored in that single model and all theories tested against it to increase capacity to impact a research question.
\end{abstract}

\section{Introduction}

In Human-Centric Functional Modeling both living and inanimate systems are represented as having a set of human-observable behaviours (functions). All the functional states accessible through these functions within a given domain of behaviour form a "functional state space" which the system acting in that domain moves through. As an example, the cognitive system executes reasoning and understanding processes, and as it does so it moves from one concept to another, thereby moving through a space of concepts or a "conceptual space" (the functional state space of the cognitive system) [1]. Using this same approach the consciousness can be represented as navigating through an "awareness space". Similarly, other living processes such as homeostasis, reproduction, and evolution can be represented as moving through their own functional state spaces. Any dynamically stable system with a stable set of repeatable functions also must stay within a bounded region of a "fitness space" that describes the fitness of the system to execute its functions. A change in fitness of the system occurs as a result of some action, that is, some path, in functional state space. In this sense the system must solve the problem of ensuring that its motion in fitness space is stable globally throughout the fitness space, despite potentially being chaotic in functional state space due to random interactions with the environment. Such systems are "adaptive problem-solving systems" because they are able to dynamically adapt in a potentially chaotic way in functional state space in order to keep their dynamics within a stable range in fitness space [2]. 
In the case of living processes, each adapts to solve problems in its own functional domain, where problems are defined as the lack of a path from an initial point in its functional state space to a final target point in that space. Solutions are defined as paths which accomplish that navigation. It has been hypothesized that every system can be considered to be a network [3]. Consider any network of functional states to be a graph containing a network of nodes, with each node representing a functional state, and with the edges or connections between functional states representing the behavior through which the system can transition from one functional state to another. This graph of the network of states accessible through these behaviors is a representation of the externally observable behavior of the system as reflected in the "functional state space". However, it does not represent the internal functions of the adaptive problem-solving system which explains the cause of that behavior, an important point to remember when applying Human-Centric Functional Modeling to physics. Representing systems as such graphs permits network analysis of the external behavior of the system in terms of its processes, their inputs, and their outputs, also called input-output analysis [4]. Because of its basic assumptions about component interconnections, this network analysis can and has been applied to many fields in which a system can be idealized as a network of interacting parts [13].

In the case of cognition the graph of conceptual space provides a representation of the meaning of each concept in terms of the reasoning processes that define it (that connect to it), and it provides a representation of the meaning of each reasoning process in terms of the concepts it connects. These meanings must be completely self-contained in the graph of conceptual space in that each cannot require any lookup tables or other external information outside that graph if the representation of the graph to be invariant across different scales. That is, a lookup table that is outside the representation of a tiny concept occupying one part in $10^{\mathrm{x}}$ of a conceptual space might not be outside the representation of a very large concept occupying one part in 10 of that conceptual space. So no lookup table at all is possible. Otherwise any representation of a concept would not then be scale invariant.

A completely self-contained representation of human meaning is a complete semantic representation. Other approaches have attempted to define complete semantic representations [5]. Where they differ is in lacking a complete model of the functions of cognition against which the statement "a complete representation of human or cognitive meaning" can be defined. In this way, through defining a complete model of the functions of cognition, and therefore of the functionality required for a representation of the meaning of a concept or of reasoning to be complete, HCFM is believed to define the requirements for a graph of conceptual space to provide a complete semantic representation of reasoning and concepts, potentially the first complete semantic representation in existence. If it can be assumed that the graph of functional state space similarly provides a complete representation of the meaning of the functional states of every other system, as well as a complete representation of the meaning of the processes by which one functional state might transition to another, then functional state space is a complete semantic representation of the functional states and processes of all other systems as well. If in addition the entire universe can be represented as a functional state space, this also implies that functional state space defines a semantic model of the physical universe. However, some properties of that conceptual space (like distances), and therefore the equivalent to those distances in all other functional state spaces, remain to be defined precisely. Without these distances, for example, all the properties of cognition whose definitions depend on those distances, as well as all the analogous properties in other functional state spaces that depend on their own distances, can't be evaluated. This set of issues is referred to here as the "representation problem".

A key function of Human-Centric Functional Modeling is to enable the navigation of all functional state spaces representing systems within the human organism as well as those functional state spaces representing inanimate systems outside the human organism, to be understood through analogy with the 
navigation of the conceptual space by the cognitive system. In this sense, Human-Centric Functional Modeling is a mathematical expression of ancient existential traditions which hold that we can only understand this existence through its impact on this human system, and that by increasing our awareness of this system, we can increase our understanding of the universe through looking inward. In the case of the "awareness space" that represents the behavior of the consciousness, this ability to look inward to our cognitive system is critical, since according to this model only behavior in the conceptual space can be inherently conceptualized and therefore rationally understood. One might assume that gaining an understanding of the functions of any system requires the behavior of that system to be conceptualizable or requires an analogy to exist between that system and one that is conceptualizable. The importance of making this analogy between consciousness, the behavior of which cannot always be conceptualized, and cognition as a system that inherently can always be conceptualized, is that it allows us to hypothesize mathematical definitions of experientially observable properties of consciousness from their similarity with properties of the cognitive system that we can mathematically deduce. As an example, general problem-solving ability (intelligence) in the cognitive domain is hypothesized to be the volume of conceptual space that can be navigated per unit time, multiplied by the density of concepts in that space, and provides a measure of the mind's ability to direct itself to a concept that solves any general conceptual problem (any problem of conceptual "fitness" which we might perceive as cognitive well-being). By analogy, general problem-solving ability in the consciousness domain might be expected to be the volume of awareness space that can be navigated per unit time, multiplied by the density of awarenesses in that space, and might be expected to provide a measure of the ability of the consciousness to direct itself to an awareness that solves any general problem of conscious well-being. This might hold for all systems in general; the importance of making the analogy between cognition and any other system is that it makes the behavior of that system conceptualizable in new ways that might provide deep insights about that system.

Having described the representation problem, the main goals of this paper and of Human-Centric Modeling Framework with regards to physics are:

\section{Goals of Extending Human-Centric Functional Modeling to Physics}

Defining the "representation problem" and the value proposition in solving it with regards to physics so that mathematical physicists, other physicists, and other experts in defining abstract problems who are interested in the study of the laws governing this physical world can be engaged to do so.

Enabling the solution to the representation problem to be used to facilitate biomimicry of the human organism in terms of replicating nature's use of the "organism pattern" as well as other patterns to optimize solutions over any collective functional state space, in order to solve wicked problems involving groups in any domains from physics to sustainable development [12].

Enabling the achievement of convergence towards a single model of physics that is most fit in reflecting all collective observations of the behavior of this universe.

Table 1: Goals in extending Human-Centric Functional Modeling to physic.

\section{The Universe as an Adaptive Problem-Solving System}

Functional state spaces can be used to represent matter in any region of the physical universe just as easily as they be used to represent the behavior of consciousness or cognition [3]. Just as human cognition can be represented as navigating a conceptual space in order to compute reasoning, the universe can potentially be represented as navigating a distributed functional state space of physical states and the forces they interact through (metaphorically the "mind" of the universe), in order to compute (metaphorically to "reason through") the evolution of matter. Just as with the conceptual space, this functional state space is represented as a graph containing a network of nodes connected by edges. But in the real universe, awareness of interactions doesn't spread at an infinite rate. Just as 
human consciousness can be represented as navigating an awareness space and in doing so can be represented as computing the movement of awareness, the universe can also potentially be represented as navigating a distributed functional state space of awareness states and the awareness processes they interact through (metaphorically the "consciousness of the universe"), in order to compute the evolution of the awareness that allows two states to interact. In other words, the universe can be represented in terms of at least two adaptive problem-solving systems, a distributed universal consciousness, and a distributed universal cognition. Since the fitness spaces that act as constraints defining the behavior of physical systems in these spaces inherently contain some variation in fitness, and therefore some uncertainty in the state of the system, the equations describing such systems can potentially be chosen to be consistent with the laws of quantum mechanics on small scales [14]. Since those fitness spaces also have the capacity to describe fitness at representing any behavior at all, they can also be chosen to be consistent with general relativity on cosmological scales.

\section{Adaptive Problem-Solving Systems Must Exceed Their Boundaries}

In Human-Centric Functional Modeling a "system" is defined as having behavior that is confined to a single functional state space in which all behaviors map from and map to a domain object in the same category. Since all functional states are represented by that same object within the functional domain in which the system operates, these states together form a space. It's important to note that these are dynamic spaces to which new states can be added and from which existing states might be removed.

In any case, whether talking about the physical universe or about biological systems, if systems adapt to external events by navigating their functional state spaces, and if functional states are continually being created in those space, there will be some finite probability that new behavior will occur outside the functional state space of the current system. If perturbations to the current system are of the right magnitude and occur over a sufficient length of time, it is hypothesized that the behavior outside the current system must increase to the point that a new domain of stability occurs, which is marked by the appearance of a new network of functional states constructed with some sequence of operations in a small set that "spans" the space. This new network of functional states represents the formation of a new system [15].

\section{Adaptive Problem-Solving Systems Must Give Rise to Self-Similar Adaptive Problem-Solving Systems}

Assume that all the potentially complex behaviors in a given domain can be represented as some combination of a very simple set of behaviors (functions or operations). In other words, assume that any network of functional states can be constructed with some sequence of only a few different types of operations. That set of operations is said to "span" the space. For example, it has been hypothesized that a set of four specific operations can "span" the conceptual space [1], and that some set of four generalized operations can potentially span any such open network of functional states [1]. Further exploration is required to determine whether this is consistent with the fact that a set of four observed physical forces appears to describe all interactions in this observable universe [?].

In addition to systems being able to potentially access all of their behaviors through using a minimal set of operations to ensure they can access their entire functional state space representing those behaviors, systems must also be able to access interactions with other systems. For example, we can confirm that our cognitive system can interact with our perception of physical sensations or emotions because it can conceptualize them. We can confirm that our consciousness can interact with our perceptions of physical sensations, emotions, or concepts, because we can become aware of them. 
In biological organisms it is hypothesized that any interaction of a system with the external environment will cause that system to experience perturbations that exceed the boundaries of its functional state space, and that where these perturbations are large enough and persist for a long enough time they will cause a sufficient volume of space to be explored outside that functional state space in order to discover a new set of processes that span a new functional state space [15]. Where this new functional state space provides lower energy states, discovering a set of processes spanning a new functional state space creates an exponentially greater number [6] of such lower energy states that are also contiguous. The exponentially greater volume of contiguous lower energy states represented by the functional state space of a new "adaptive problem-solving system" suggests that such new systems form a stable potential energy well which part of the entity might come to continuously occupy. This new system might be a "parent" system if its functional state encompasses the functional state of the original system, similar to the way that concepts as the functional states of the cognitive system can encompass (can include and therefore conceptualize) sensory perceptions or emotions. Alternatively this new system might be a "sibling" system if its functional state does not encompasses the functional state of the original system, such as visual perceptions as the functional states of the visual system don't encompass (don't include and therefore can't represent) tactile or other sensory perceptions.

Once a new system is formed, we might assume those processes must be somehow cyclic, since cyclic processes maximize the production of entropy [7], [8]. This supports the hypothesis that cyclic dynamics represent adaptive problem-solving solving systems in fitness space. That is, the fact that energy is continually being added to such systems combined with the requirement that the dynamics of adaptive problem-solving systems be globally stable in some fitness space suggests that those dynamics are cyclic.

As mentioned, any model hypothesized to represent all systems must show how one system might be able to interact with another external system, as real systems are observed to do. Representing physical sensations, and emotions as three dimensional fields of signals, it is hypothesized that only three functions are required for the cognition to conceptualize (that is, to interact with) any sensory or emotional signal in those fields. Representing concepts as forming such a field as well, it is hypothesized that the same three functions can be used by the consciousness to interact with (to become aware of) any sensory, emotional, or conceptual signal in those respective fields. The point is that the same mechanism by which systems might interact might work between any two systems represented in functional state space.

\section{Applicability to Representing the Universe as a Hierarchy of Functional State Spaces}

Assume that the universe arose from a much simpler system with a much simpler set of states and different interactions between those states (different forces) than we see in our current universe. Assume our current universe might in fact be represented by a hierarchical network of functional state spaces just like the human organism is represented by a hierarchical network of functional state spaces [2]. Functional state spaces describe the externally observable behaviors of systems. The internal behavior of systems determines the dynamics with which systems navigate those functional state spaces, and therefore determines the processes by which systems might transition from one functional state to another, or put a different way, the internal behavior of systems determines the interactions between functional states. In "constructor theory" [9], [10] the internal behavior of systems can be represented as a "constructor" containing the laws that determine the fitness of any processes to describe the observed behavior of the system. In Human-Centric Functional Modeling the internal behavior of any adaptive problem-solving system is represented as a constructor that generates the external behavior of the system. 
In the same way that homeostasis might represent a cyclic process that maximizes the production of entropy within a potential energy well, creating stable dynamics that allowed the earliest life to exist, and the same way that over time perturbations of that homeostasis process by the external environment might have led to exceeding the boundaries of those stable dynamics to create new systems such as cellular reproduction, cellular growth, or others, the physical universe might have begun through some very simple system with stable dynamics, perhaps created by a single property distributed nonuniformly throughout space, and that system might have spread.

Defining the simplest possible universe, such as one as having a non-uniformity in a single property, then representing that universe in terms of a functional state space, the more complex universes that might arise from that universe might also be represented as functional states spaces, so that our current universe then becomes the sum of those functional state spaces. Without even having to know what that initial property was, through defining the simplest possible functional state space that might represent such a property, through deducing how new spatially invariant properties might arise through simple geometric transformations of that property, and how new spatially invariant properties might arise through simply geometric transformations of those properties in turn, and so on, it might be possible to construct this universe from a set of simpler ones as some have attempted to do [11]. And collaboratively it might be possible to methodically explore a far wider set of such universes to more reliably arrive at one that is most fit at representing the observed properties of this physical universe, as well as being most fit at representing features of the universe like dark matter that can only be described through limited interactions with this universe, like the gravitational interaction.

Consider that all geometric transforms can be seen as processes of generalization. For example, all circles and ellipses are solutions of the same general equation to which all the geometric transforms can be applied. Truth can be defined in two ways. Relative truth is a signal. Any given reasoning processes in conceptual space, or any given process in any other functional state space, is a channel that conveys truth with some degree of error that is given by information theory [6]. In other words, in conceptual space the relative truth of one claim can be defined in terms of the self-consistency of the group of other claims contained in the generalization of that claim. In addition, any geometry in any Euclidean or positively or negatively curved space is hypothesized here to represent an absolute truth, but whether all truths can be represented by some geometry has not been proved [16].

Once relative or absolute truths can be defined in any functional state space then relationships can be defined between those truths. Defining absolute or relative truths as being related by such transforms enables any reasoning that is "true" in terms of being a solution of any one problem to be generalized through those transforms to any other problem where it applies (where it is also true). As another example, Human-Centric Functional Modeling represents the conceptual space belonging to a given instance of cognition as having some regions that might be occupied by concepts, and some regions that might be empty. Valid properties of concepts have some degree of invariance in that the reasoning relationships defining those properties belong to that concept. These relationships might be translated, reflected, rotated, etc. (that is, geometric transforms might be applied) to reveal new invariant relationships that apply to concepts in other regions of conceptual space as well.

In the same way that properties of concepts must be invariant in conceptual space if those properties are to belong to the concepts, properties of matter or energy must belong to matter or energy, that is, though they might change and therefore be different at different places and times, they must not depend on any particular place or any particular time. In other words they must be invariant with respect to space and time, as opposed to being properties of space and time. As an example, the position of a 
particle in space can't define a fundamental property of the particle. Because that position can have any value without changing the particle.

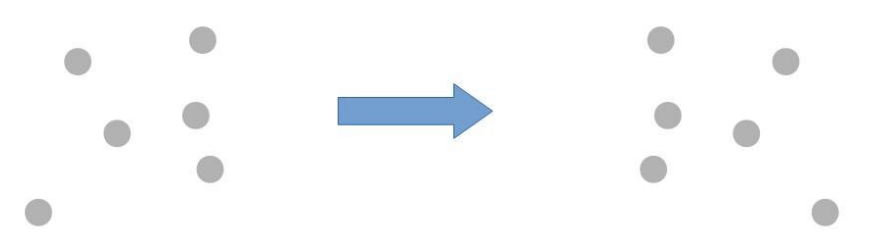

Figure 1: A reflection by itself is not an invariant property of the set of regions.

However, multiple entities can interact, and that interaction can have geometrical properties in space and time that are in fact invariant. Through using functional state space to define and explore all possible interactions between non-uniform distributions in a property through space and time, and through defining and exploring all the geometric transforms through which those interactions can be mapped to all other interactions, it becomes possible to enumerate all of the spatially and temporally invariant interactions that might potentially form invariant properties of any entity such as matter.

Even if we can't confirm that our functional model can represent all behaviors observed in matter and energy within this universe, if we can validate that assuming a finite propagation time for interactions, assuming a non-uniform distribution of a property, and assuming an interaction of those non-uniform distributions on some geometric scale, that this functional modeling approach can represent all possible interactions, then these interactions in combination with these simple transforms can at least be confirmed to represent a wide range of observed behaviors. This is true because the geometrical transforms can introduce any number of new interactions, which together might represent the observed fundamental forces and the properties of matter and energy.

As one example, the translational interaction of two non-uniform regions might result in rotation. That rotational interaction might propagate at a speed characteristic of speed of the "awareness interaction" because any translational motion must result in one region moving a certain distance before the other becomes aware of its new position, and then the second moving a certain distance before the first becomes aware of its new position. This characteristic speed then defines the highest speed possible, potentially related to observable properties like the speed of light. In addition, the pair must move in a way that conserves linear and forward momentum, but there might be an angular velocity characteristic of the interaction strength, range, and other properties. By choosing the interaction strength and range, it may be possible to introduce an invariant property of angular velocity that might conceivably be related to the observable property like "spin". If so, as explained below, this angular velocity will be the maximum attainable. All other interactions will have an angular velocity that is some fraction of this. 


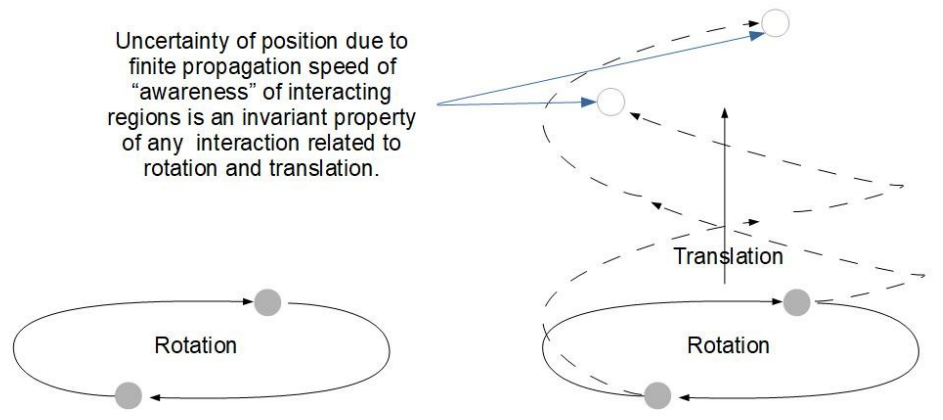

Figure 2: Any rotational interaction (left) can potentially involve some translation (right). This combination of transation and rotation can define an invariant property of the interaction due to the finite propagation speed of the interaction.

The translational interaction of more than two non-uniform regions might on the other hand be chaotic, even if the interaction has some net rotation, because in stable solutions to the N-body problem those bodies likely don't have angular velocities that are aligned.
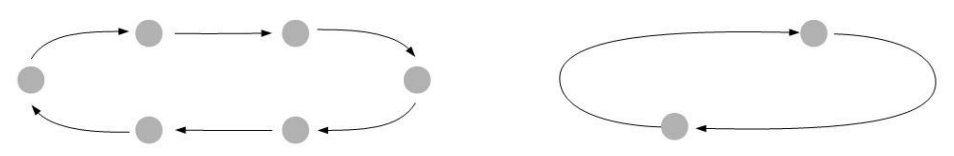

Figure 3: $N$-body rotation ( $N$ greater than two) with the angular velocity of all bodies aligned into a single rotation (left) might not be stable. Two-body rotation (right) can be demonstrated to be stable.

For this reason, rotational interaction of multiple-non uniform regions may be constrained to a lower angular velocity than that of two non-uniform regions. As a consequence we might expect to see angular velocity constrained to certain values. Computationally exploring interaction characteristics, with the right interaction strength and range that angular velocity could potentially converge on some number observed to be characteristic of matter. In addition, a translational interaction of these $\mathrm{N}$ nonuniform regions ( $\mathrm{N}$ greater than two) because of its more complex motion, could in turn result in that interaction propagating at a speed slower than the characteristic speed of the "awareness" because with such complex motion the group cannot coherently move in a single direction. This would reduce the propagation speed so the velocity could never exceed the characteristic speed of non-uniformities. This might conceivably be related to the observation that the maximum speed of matter appears to be the speed of light. 


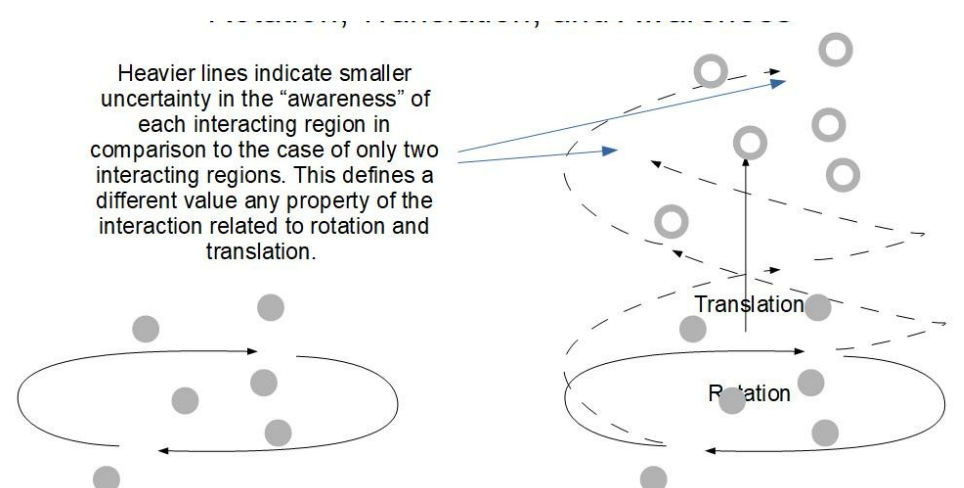

Figure 4: In a larger set of regions any rotational interaction (left) might again involve translation (right). Due to lower uncertainty in the position of the interaction in the case of of larger numbers of regions, this combination of translation and rotation can define a different value of any invariant property of the interaction that arises due to the finite propagation speed of the interaction.

In the case of more than two non-uniform regions, one in the set will interact with the second in the set through its awareness of the state of the second, and will interact with the third through its awareness of the state of the third, and so forth to the Nth. Because awareness of this net state is potentially a far larger constraint, it could give groups of such non-uniformities a kind of "inertia" and therefore "mass". In the same way that forcing water through a pipe too fast causes resistance, forcing too much information through the exchange of this awareness could potentially form a repulsive force so that such masses resist occupying the same space. In the case that relative values of some other property (like angular velocity being aligned) acts to increase or decrease that repulsive force, this force could become attractive such as the case with opposite charges, or repulsive in the case of like charges.

As another example of potential interactions, a deformation is a pattern in the non-uniformity. This pattern is a non-uniformity in the non-uniformity. Such an interaction might enable "dimensions" to interact.

Figure 5: Uncertainty of position due to finite propagation speed of "awareness" of interacting regions is an invariant property of any scaling interaction.

An interaction that is scaled to include a number of regions might create a significantly larger constraint on "awareness". If the non-uniformities represent energy, then this means that scaling interactions, such as by composing a larger interaction from a number of component interactions, might significantly increase energy in the same way that each subatomic particle increases the energy of an atom.

An interaction that deforms a region might also introduce some invariant property. 


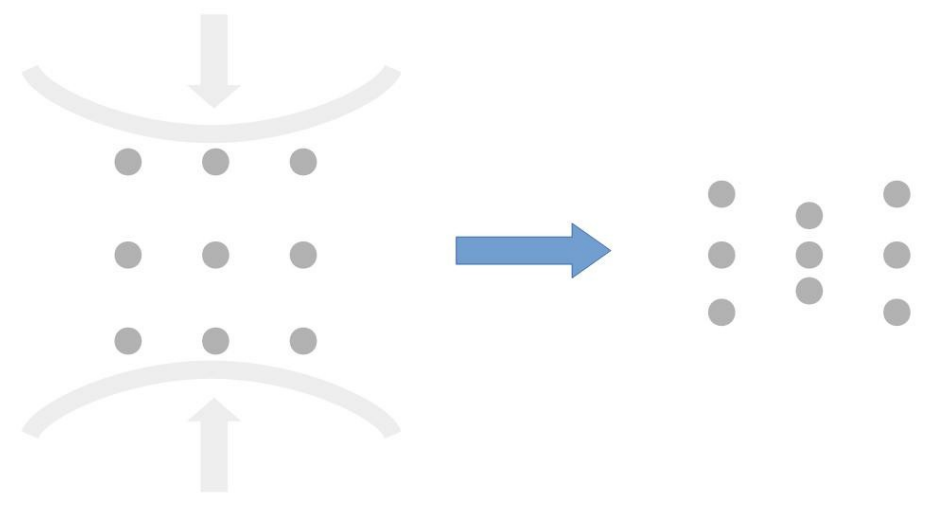

Figure 6: A deformation interaction might also define an invariant property.

In summary, these speculative examples are not meant to suggest new physics but only to demonstrate that each of a great many universes might be represented by its own functional state space and these functional state spaces might interact. Any countless combination of such universes might be explored through computer simulation. The boundary conditions for our functional model are that in whatever dimension " $\mathrm{N}$ " in this model that represents the observed universe, the sum of all the interactions between the functional state spaces represented in this model must reduce to the observed properties of matter and energy.

\section{Conclusion}

A key goal of Human-Centric Functional Modeling is to create a vastly improved ability to converge on whatever functional model is most fit at representing the observed functions of this universe. There are already a number of models that have been proposed. Rather than just defining another, Human-Centric Functional Modeling begins by defining a modeling approach with which one might determine whether a model can be confirmed through first person observation to be complete and self-consistent. In the current state of affairs each model of a unified theory in physics is championed by a small number of individuals. Given that developing and/or understanding any one particular meta-model of can be a life-long achievement, and assuming that understanding and comparing more than one or two such meta-models might be outside the cognitive capacity of most individuals, one potential key area of usefulness for Human-Centric Functional Modeling is that it provides a single universal way of modeling the physical universe so that all such meta-models might be compared in each context in order to collectively converge on a single meta-understanding of physics that best reflects observations. Implementing and validating any single model of such a meta-theory is not a goal that can reliably be accomplished by any single investigator or small group of investigators, since the number of entities that must be modeled and implemented far exceeds the capacity of any single investigator or small group of investigators. However, if a universal approach such as HCFM towards representing all models can be defined, and if the problems that must be solved to implement such a model can be decoupled so that each individual only has to understand the value proposition of this approach for their research alone, while at the same time the underlying symmetry between the problems can be used to scale the value created with the number of participants, then the value created by this research might be scaled until the required participants can reliably be engaged. It's hoped that this article will inspire others to independently confirm or refute the viability of this model as well as its predictions.

\section{References}


[1] Williams A.E. (2020) A Model for Artificial General Intelligence. In: Goertzel B., Panov A., Potapov A., Yampolskiy R. (eds) Artificial General Intelligence. AGI 2020. Lecture Notes in Computer Science, vol 12177. Springer, Cham. https://doi.org/10.1007/978-3-030-52152-3 38

[2] Andy E. Williams, The Architecture of Cognition as a Generalization of Adaptive Problem-Solving in Biological Systems, 2021 Conference on Biologically Inspired Cognitive Architectures (BICA), Accepted.

[3] Dodig Crnkovic, G. Dynamics of Information as Natural Computation. Information 2011, 2, 460477. https://doi.org/10.3390/info2030460

[4] Leontief, W.W., 1966. Input-Output Economics. Oxford University Press, New York, NY.

[5] Lewis, Martha, Dan Marsden, and Mehrnoosh Sadrzadeh. "Semantic Spaces at the Intersection of NLP, Physics, and Cognitive Science." FLAP 7.5 (2020): 677-682.

[6] Williams, A. E. (2021, October 2). Using Systems Thinking to Exponentially Increase Impact. https://doi.org/10.31730/osf.io/dcjyf

[7] MAURER, B.A. \& D.R. BROOKS. 1991. Energy flow and entropy production in biological systems. J. Ideas 2: 48-53.

[8] Martyushev, L.; Seleznev, V. Maximum entropy production principle in physics, chemistry and biology.Phys. Rep.2006,426, 1-45

[9] Deutsch, David. "Constructor theory." Synthese 190.18 (2013): 4331-4359.

[10] Marletto, Chiara. "Constructor theory of life." Journal of The Royal Society Interface 12.104 (2015): 20141226.

[11] Wolfram, Stephen. "A class of models with the potential to represent fundamental physics." arXiv e-prints (2020): arXiv-2004.

[12] Williams, A.E. (2021c) Are wicked problems a lack of general collective intelligence?. AI \& Soc (2021). https://doi.org/10.1007/s00146-021-01297-8

[13] Solé, Ricard, and Sergi Valverde. "Evolving complexity: how tinkering shapes cells, software and ecological networks." Philosophical Transactions of the Royal Society B 375.1796 (2020): 20190325

[14] Andy E. Williams, Emir Haliki, Human-Centric Functional Modeling and Condensed Matter Physics, working paper (2021), Retrieved from osf.io/preprints/africarxiv/2hwtv

[15] Andy E. Williams, Human-Centric Functional Modeling and Evolutionary Biology, in review (2021), Retrieved from osf.io/preprints/africarxiv/sy4b6

[16] Andy E. Williams, Comment in Reply to "On the complexity of extending the convergence region for Traub's method", working paper (2021), Retrieved from osf.io/preprints/africarxiv/2zbyq 
[17] Chaichian M., Perez Rojas H., Tureanu A. (2021) Four Fundamental Forces. In: Basic Concepts in Physics. Undergraduate Lecture Notes in Physics. Springer, Berlin, Heidelberg.

https://doi.org/10.1007/978-3-662-62313-8_9 